\title{
Bullous hemorrhagic dermatosis
}

\author{
Simon F. Roy MD, Philippe Watson MD, Danielle Bouffard MD
}

Cite as: CMAJ 2020 January 6;192:E12. doi: 10.1503/cmaj.191052

A

52-year-old woman presented with a 4-day history of painful, tense, blood-filled blisters on her ankles and dorsal feet, with no mucosal involvement (Figure 1). She had a history of plurimetastatic carcinoma of likely pancreatobiliary origin, for which she had received 2 cycles of immune-activating monoclonal antibodies (durvalumab and tremelimumab) 2 months earlier. For the past 5 months, she had also been receiving a therapeutic regimen of tinzaparin (16000 units/d subcutaneously) for treatment of pulmonary embolism. Tinzaparin had been temporarily replaced with enoxaparin for an 8-day span, as it was unavailable at the hospital, 2 weeks before the eruption of blisters.

Our differential diagnosis included bullous leukocytoclastic vasculitis or varicella-zoster virus infection. Her complete blood count showed a white blood cell count of $10.2 \times 10^{9} / \mathrm{L}$, a hemoglobin level of $94 \mathrm{~g} / \mathrm{L}$ and a platelet count of $154 \times 10^{9} / \mathrm{L}$. Prothrombin time, partial thromboplastin time and international normalized ratio were normal (12 s, $28 \mathrm{~s}$ and 1.09, respectively). Histopathologic examination of one of the blisters showed a broad hemorrhagic subepidermal bulla, with no inflammation (excluding leukocytoclastic vasculitis) or viral cytopathic effect (excluding varicella-zoster virus). Although immunotherapy agents may precipitate blistering disorders, the timeline and absence of inflammation on biopsy made this explanation unlikely.

Clinical and histopathologic findings were consistent with bullous hemorrhagic dermatosis, an adverse drug effect probably induced by subcutaneous low-molecular-weight heparin. It is unclear whether re-initiation of tinzaparin or the previous temporary course of enoxaparin was responsible in the case of our patient. Bullous hemorrhagic dermatosis tends to arise after a mean of 7 (range 3-270) days of starting the drug. ${ }^{1}$ It may also occur with use of dalteparin, warfarin, fondaparinux and unfractionated heparin. ${ }^{1}$ Changing anticoagulant therapy does not hasten lesion resolution as the condition is self-resolving, hence the importance of maintaining the current anticoagulation treatment if still indicated. ${ }^{1}$ Our patient's lesions resolved within 3 weeks, with the same anticoagulation regimen. Unfortunately, she died 4 months after presentation from metastatic disease.

\section{Reference}

1. Russo A, Curtis S, Balbuena-Merle R, et al. Bullous hemorrhagic dermatosis is an under-recognized side effect of full dose low-molecular weight heparin: a case report and review of the literature. Exp Hematol Oncol 2018;7:15.

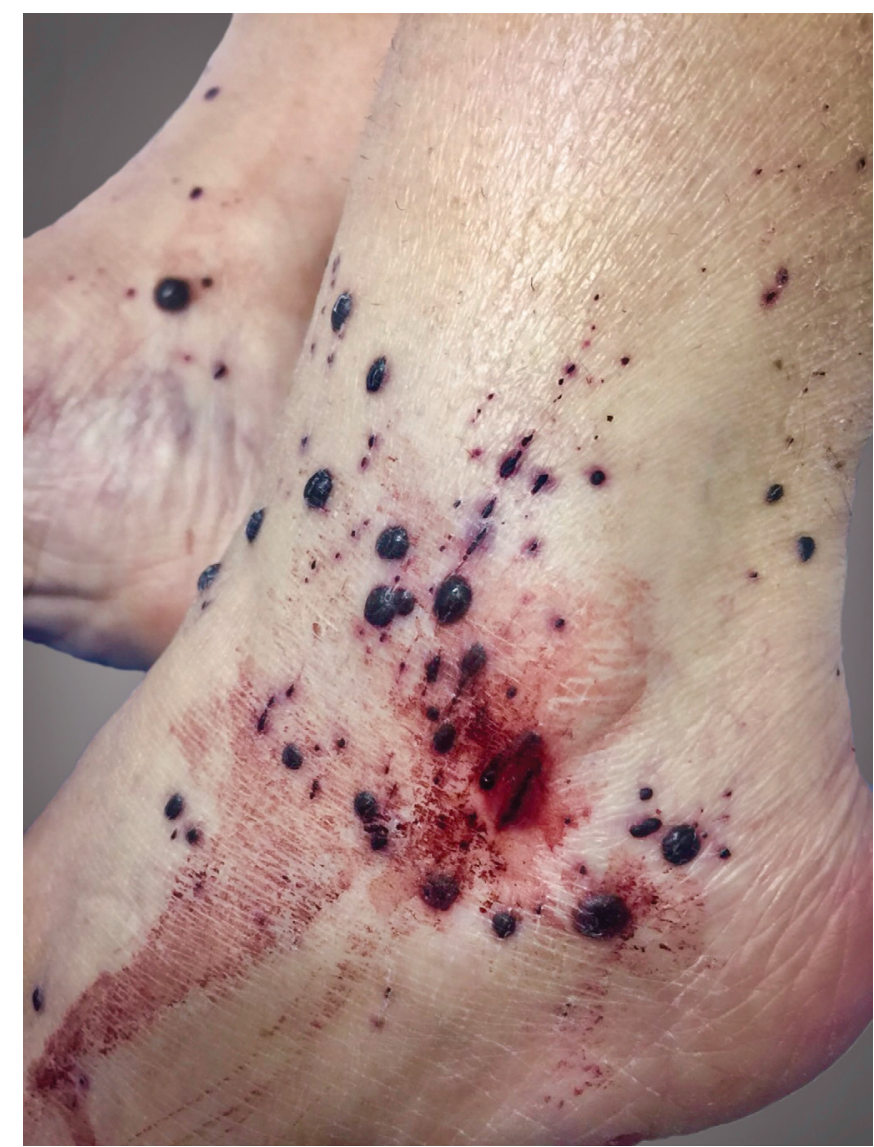

Figure 1: Multiple tense, hemorrhagic blisters, some of which have ruptured, on the ankles and dorsal feet of a 52-year-old woman receiving treatment with low-molecular-weight heparin.

\section{Competing interests: None declared.}

This article has been peer reviewed.

The authors have obtained patient consent.

Affiliations: Divisions of Pathology (Roy, Bouffard) and Dermatology (Watson), Université de Montréal, Montréal, Que.

Correspondence to: Danielle Bouffard, daniellebouffard@hotmail.com 